Article

\title{
Synthesis and Antitumor Activity of 5-Bromo-7-azaindolin-2-one Derivatives Containing a 2,4-Dimethyl-1H-pyrrole-3-carboxamide Moiety
}

\author{
Jun Zhang ${ }^{1,2,+}$, Weiyi Shen ${ }^{2,+}$, Xiaoning Li ${ }^{1}$, Yun Chai ${ }^{1, *}$, Senjun $\mathrm{Li}^{2}$, Kai Lv ${ }^{1}$, Huiyuan Guo ${ }^{1}$ \\ and Mingliang Liu ${ }^{1, *}$ \\ 1 Institute of Medicinal Biotechnology, Chinese Academy of Medical Sciences and Peking Union Medical \\ College, Beijing 100050, China; jun0215@126.com (J.Z.); 1xn18273645@163.com (X.L.); \\ lvkailk@hotmail.com (K.L.); imbhyguo@126.com (H.G.) \\ 2 Zhejiang Starry Pharmaceutical Co. Ltd., Xianju 317300, China; \\ swy109@starrypharma.com (W.S.); lisj0966@163.com (S.L.) \\ * Correspondence: yunchai18@126.com (Y.C.); lmllyx@126.com (M.L.); \\ Tel.: +86-10-6316-5280 (Y.C.); Tel./Fax: +86-10-6303-6965 (M.L.) \\ + These authors contributed equally to this work.
}

Academic Editor: Maria Emília de Sousa

Received: 11 November 2016; Accepted: 30 November 2016; Published: 6 December 2016

\begin{abstract}
We report herein the design and synthesis of a series of novel 5-bromo-7-azaindolin-2-one derivatives containing a 2,4-dimethyl- $1 \mathrm{H}$-pyrrole-3-carboxamide moiety. These newly synthesized derivatives were evaluated for in vitro activity against selected cancer cell lines by MTT assay. Results revealed that some compounds exhibit broad-spectrum antitumor potency, and the most active compound $23 \mathrm{p}\left(\mathrm{IC}_{50}: 2.357-3.012 \mu \mathrm{M}\right)$ was found more potent than Sunitinib $\left(\mathrm{IC}_{50}: 31.594-49.036 \mu \mathrm{M}\right)$ against HepG2, A549 and Skov-3, respectively.
\end{abstract}

Keywords: 5-bromo-7-azaindolin-2-ones; synthesis; antitumor activity

\section{Introduction}

Sunitinib (Figure 1) is a new multitargeted oral anti-angiogenic and antitumor drug that has been recently approved against gastrointestinal stromal tumors (GIST) and advanced renal cell carcinoma (RCC) [1]. It is in clinical studies for the treatment of other solid tumors, such as pancreatic neuroendocrine tumors [2], meningioma [3], metastatic breast cancer [4] and non-small cell lung cancer [5].

Recently, structural modifications mainly at the 3- and 5-positions of the indolin-2-one ring of Sunitinib have made considerable progress in the ability to increase antitumor activity through inhibition on different receptors [6-8]. As early lead compounds discovered in our lab, Z24 and LK-B030 (Figure 1) bearing a (piperidin-1-yl)methyl and a (3-dimethylamino)propyl group at the N-1 position, respectively, display a broad spectrum of antitumor activity by inhibiting angiogenesis in new blood vessels [9-11]. More recently, we reported a series of novel 5-halogenated-7-azaindolin-2-one derivatives and found IMB-1501 to have better in vitro activity than Sunitinib against the entire tested cancer cell lines [12].

As part of our continuing modifications on Sunitinib as a potential antitumor drug candidate, we planned to explore other possibilities for diversification of the 2-(pyrrolidin-1-yl)ethyl group and the linker flexibility on the amide bond. Thus, a series of novel 5-bromo-7-azaindolin-2-one derivatives containing a 2,4-dimethyl- $1 \mathrm{H}$-pyrrole-3-carboxamide moiety were designed, synthesized and evaluated for their antitumor activity in this study. Our primary objective was to optimize the potency of these compounds against a set of solid tumors and contribute to the development of 
new antitumor agents. A preliminary structure-activity relationship (SAR) study is also explored to facilitate the further development of 5-bromo-7-azaindolin-2-ones.
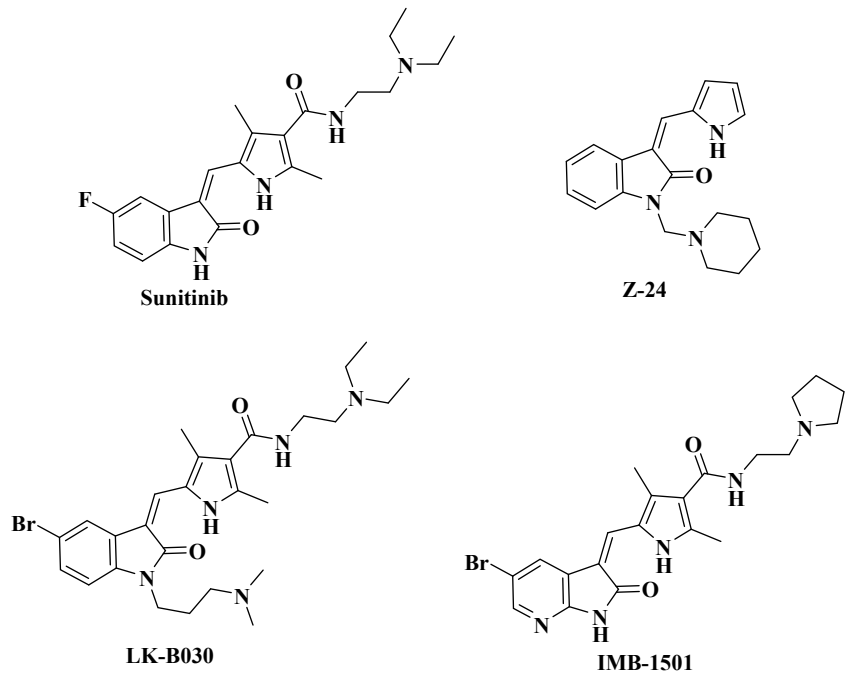

Figure 1. Structures of Sunitinib, Z-24, LK-B030 and IMB-1501.

\section{Results and Discussion}

Detailed synthetic pathways to heterocyclic amine derivatives 5-7 and 14-16, which are commercially unavailable, and target compounds 23a-q are depicted in Schemes 1-3, respectively. Amine derivatives containing aminoalkyl groups 5-7 were easily obtained from pyrrolidine 1, piperidine 2 and $\mathrm{N}$-methylpiperazine 3 by nucleophilic substitution with $\mathrm{N}$-(2-bromoethyl)/ $\mathrm{N}$-(3-bromopropyl) $/ \mathrm{N}-(4-$ bromobutyl)phthalimides $4 \mathrm{a}-\mathrm{c}$ in the presence of $\mathrm{K}_{2} \mathrm{CO}_{3}$ in DMF at $70-80{ }^{\circ} \mathrm{C}$, respectively, followed by treatment of the resulting condensates with hydrazine hydrate in ethanol under reflux condition (Scheme 1).

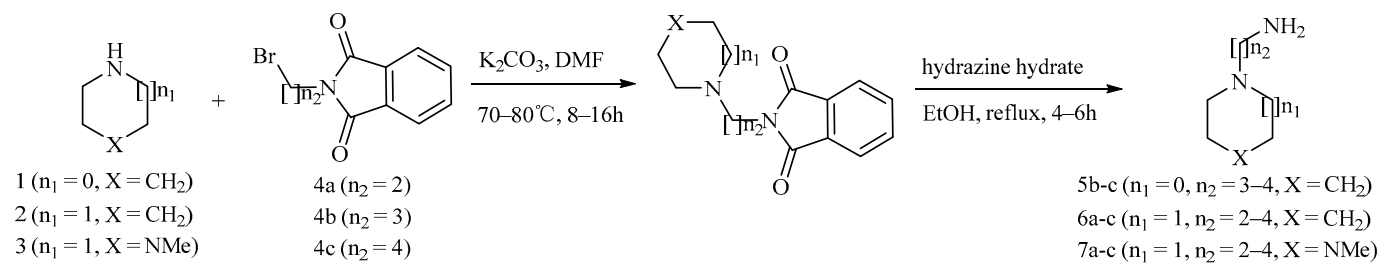

Scheme 1. Synthesis of amine derivatives 5-7.

Condensation of pyrrolidin-3-one 8, piperidin-3-one 9 and piperidin-4-one 10 with $\mathrm{O}$-alkylhydroxyamines gave compounds 11-13. Amine derivatives 14-16 were prepared from oximes 11-13 by coupling with $\mathbf{4 b , c}$ and hydrazinolysis, sequentially (Scheme 2 ).

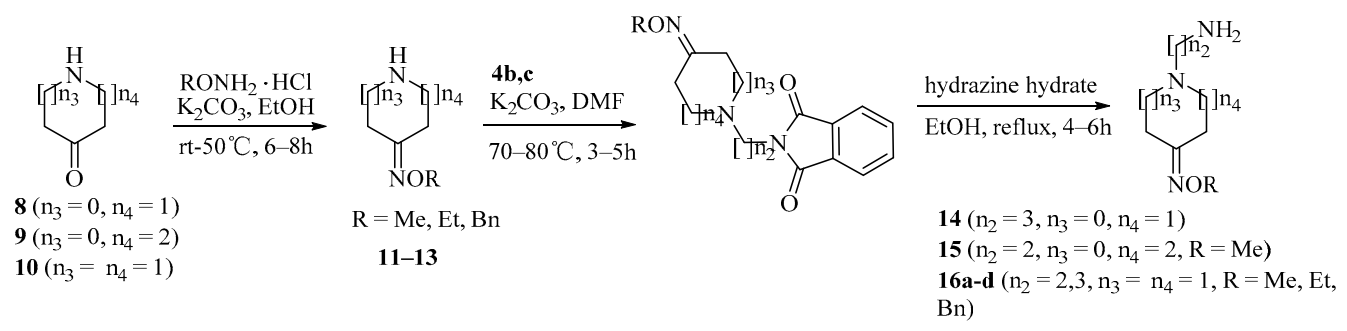

Scheme 2. Synthesis of amine derivatives 14-16. 
Amidation of 5-formyl-2,4-dimethylpyrrole-3-carboxylic acid 17 with 5-7, 14-16 and commercially available (S)-1-amino-3-morpholinopropan-2-ol (18), $N^{1}, N^{1}$-dimethylpropane-1,3-diamine (19) and $N^{1}, N^{1}$ diethylpropane-1,3-diamine (20) in the presence of 1-ethyl-3-(3-dimethylaminopropyl)carbodiimide hydrochloride (EDCI), $N$-hydroxybenzotriazole (HOBt) and Ethyldiisopropylamine (DIEA) yielded compounds 21a-q. Aldol condensation of 5-formyl-2,4-dimethylpyrrole-3-carboxamides 21a-q with 5-bromo-7-azaindolin-2-one $\mathbf{2 3}$ in the presence of piperidine gave the target compounds $\mathbf{2 3 a - q}$ (Scheme 3) [10,11]. All of the new synthetic compounds were well characterized by ${ }^{1} \mathrm{H}-\mathrm{NMR}$, ${ }^{13} \mathrm{C}-\mathrm{NMR}$ and MS. As expected, the pyrrole-2-methylidene geometry at the 3-position of the 7-azaindolin-2-one ring was confirmed to have the Z-configuration [12-14].
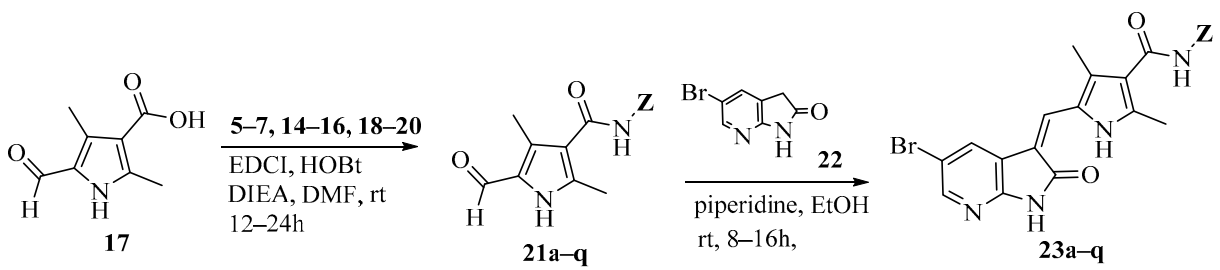

Scheme 3. Synthesis of target compounds $23 \mathbf{a}-\mathbf{q}$.

We first replaced the ethylidyne linker of Sunitinib with propylidyne or butylidyne, and the diethylamino group with a saturated heterocyclic amine (pyrrolidine, piperidine, piperazine, or hydroxylmorphine [15]) to synthesize the derivatives 23a-e. For preliminary screening of antitumor candidates, the target compounds were investigated for cytotoxic activity in vitro against MCF-7 (a breast cancer cell line). It is encouraging that all of the initially designed molecules except 23e exhibit higher inhibition $(81.46 \%-87.29 \%)$ than Sunitinib $(27.79 \%)$ at the concentration of $30 \mu \mathrm{M}$ (Table 1). They were further evaluated for their in vitro antitumor activity in six human cancer cell lines, including MCF-7, HepG2 (liver carcinoma), HT-29 (colon adenocarcinoma), A549 (lung adenocarcinoma), PANC-1 (pancreatic carcinoma) and Skov-3 (ovarian carcinoma) by MTT assay [16].

Table 1. In vitro activity of target compounds 23a-e against six cell lines.

\begin{tabular}{|c|c|c|c|c|c|c|c|c|}
\hline & & & & $23 a-e$ & & & & \\
\hline \multirow{2}{*}{ Compound } & $\mathrm{Z}$ & \multirow{2}{*}{$\%$ Inhibition ${ }^{a}$} & \multicolumn{6}{|c|}{ IC50 $(\mu \mathrm{M})$} \\
\hline & & & MCF-7 & HepG2 & HT-29 & A549 & PANC-1 & SKOV-3 \\
\hline $23 a$ & & 87.29 & 12.790 & 7.060 & 8.893 & 4.993 & 14.132 & 5.766 \\
\hline $23 b$ & & 83.61 & 27.457 & 6.852 & 10.820 & 6.555 & 13.672 & 5.978 \\
\hline $23 c$ & & 81.46 & 17.946 & 7.882 & 9.524 & 3.103 & 9.410 & 6.669 \\
\hline $23 d$ & & 84.98 & 15.052 & 6.316 & 10.388 & 6.250 & 9.512 & 3.721 \\
\hline $23 e$ & & 46.83 & 65.054 & 36.964 & 19.642 & 26.031 & 33.314 & 29.685 \\
\hline Sunitinib & & 27.79 & 65.606 & 41.805 & 31.774 & 29.257 & 54.916 & 31.985 \\
\hline
\end{tabular}


The data reveal that 5-bromo-7-azaindolin-2-ones 23a-e demonstrate increased activity $\left(\mathrm{IC}_{50}: 3.103-65.054 \mu \mathrm{M}\right)$ compared to Sunitinib ( $\left.\mathrm{IC}_{50}: 29.257-65.606 \mu \mathrm{M}\right)$ against all of the tested cancer cell lines (Table 1). In particular, compound 23c exhibits a value of $3.103 \mu \mathrm{M}$ against A549 and 23d exhibits a $\mathrm{IC}_{50}$ value of $3.721 \mu \mathrm{M}$ against Skov-3, which are 9.4- and 8.6-fold more potent than Sunitinib, respectively. It seems likely that the linker between the amide and the pyridine ring is well tolerated with an alkyl chain of C3/C4 (23a-b). Moreover, bearing a pyridine or piperidine or piperazine moiety with an alkyl chain of C2-4 is more favorable than the introduction of a hydroxy group on the alkyl linker (23a-d vs. 23e).

Being encouraged by the above results, we further explored other possibilities for diversification of the linker or/and heterocyclic amine to design and synthesize the derivatives $\mathbf{2 3} \mathbf{f}-\mathbf{q}$ which were evaluated for their activity in selected cell lines HepG2, A549 and SKOV-3 (Table 2). When the ethylidyne linker (23c, 23d) was replaced by a propylidyne or butylidyne moiety, the resulting compounds $(\mathbf{2 3 f}, \mathbf{2 3 g}, \mathbf{2 3 i})$ were found to have better activity $\left(\mathrm{IC}_{50}: 5.023-7.803 \mu \mathrm{M}\right)$ than Sunitinib ( $\mathrm{IC}_{50}$ : 31.594-49.036 $\left.\mu \mathrm{M}\right)$. However, replacement of the butylidyne linker (23i) with a propylidyne moiety or opening of the piperidine ring (23f) led to decreased potency, although the corresponding compounds (23h, 23j, 23k) demonstrate similar activity.

Table 2. In vitro activity of target compounds $23 \mathbf{f}-\mathbf{q}$ against three cell lines.<smiles>CNC(=O)c1c(C)[nH]c(/C=C2\C(=O)Nc3ncc(Br)cc32)c1C</smiles>

\begin{tabular}{|c|c|c|c|c|}
\hline \multirow{2}{*}{ Compound } & \multirow[b]{2}{*}{$\mathbf{Z}$} & \multicolumn{3}{|c|}{$\mathrm{IC}_{50}(\mu \mathrm{M})$} \\
\hline & & HepG2 & A549 & SKOV-3 \\
\hline $23 \mathrm{f}$ & & 7.144 & 6.982 & 5.023 \\
\hline $23 \mathrm{~g}$ & & 7.803 & 6.137 & 7.507 \\
\hline $23 \mathrm{~h}$ & & 11.543 & 13.506 & 15.126 \\
\hline $23 i$ & & 7.657 & 6.254 & 6.470 \\
\hline $23 j$ & & 14.916 & 13.443 & 14.916 \\
\hline $23 k$ & & 12.229 & 10.273 & 10.998 \\
\hline
\end{tabular}


Table 2. Cont.

\begin{tabular}{|c|c|c|c|c|}
\hline \multirow[b]{2}{*}{ Compound } & \multirow[b]{2}{*}{$\mathrm{Z}$} & \multicolumn{3}{|c|}{$\mathrm{IC}_{50}(\mu \mathrm{M})$} \\
\hline & & HepG2 & A549 & SKOV-3 \\
\hline 231 & & 60.617 & 59.319 & 40.087 \\
\hline $23 m$ & & 99.667 & 119.493 & 25.090 \\
\hline $23 n$ & & 22.054 & 36.509 & 17.296 \\
\hline 230 & & 6.828 & 7.731 & 7.747 \\
\hline $23 p$ & & 3.012 & 2.357 & 2.659 \\
\hline $23 q$ & & 5.878 & 6.681 & 4.075 \\
\hline Sunitinib & & 33.999 & 31.594 & 49.036 \\
\hline
\end{tabular}

Considering the importance of an oxime functional moiety of the C-7 side chain with respect to the antibacterial and/or antitumor activity of quinolones [17-19], the impact of an alkoxyimino group on the pyrrolidine or piperidine ring was also investigated. It is clear that the introduction of a methoxyimino group on the pyrrolidine ring is detrimental (231). For the piperidine ring, the nature and position of the oxime group and linker greatly influence activity. For instance, the presence of a methoxyimino group at the para-position is more favorable than the meta-position (23n vs. 23m). In addition, the propylidyne linker (23o) displays MIC values of $6.828-7.747 \mu \mathrm{M}$, which are 2.2- to 4.7-fold more potent than the ethylidyne linker (23n). It is also shown that the methyl group of the oxime moiety (23o) could be replaced by an ethyl (23p) or a benzyl (23q) one without obviously affecting the antitumor potency. Among the three, the most active compound $23 p\left(\mathrm{IC}_{50}: 2.357-3.012 \mu \mathrm{M}\right)$ was found to be 11.3- to 18.4-fold more potent than Sunitinib against all of the tested cell lines, respectively (Table 2).

\section{Experimental Section}

Melting points were determined in open capillaries and are uncorrected. ${ }^{1} \mathrm{H}-\mathrm{NMR}$ and ${ }^{13} \mathrm{C}-\mathrm{NMR}$ spectra were determined on a Varian Inova-500 spectrometer (Varian, Inc., Palo Alto, CA, USA) in DMSO- $d_{6}$ using tetramethylsilane as an internal standard. Electrospray ionization (ESI) mass spectra were obtained on an MDSSCIEX Q-Tap mass spectrometer (AB Sciex, Redwood City, MA, USA) and Advion Mass Express 2.1.243 (Advion BioSciences, Inc., Ithaca, NY, USA). The reagents were all of analytical grade or chemically pure. TLC was performed on silica gel plates (Merck, ART5554 60F254, Kenilworth, NJ, USA).

A mixture of heterocyclic amines (1-3, $5.9 \mathrm{mmol})$, 2-(2-bromoethyl/propyl/butyl)isoindoline-1,3diones $(4 \mathbf{a}-\mathbf{c}, 7.0 \mathrm{mmol})$ and potassium carbonate $(7.0 \mathrm{mmol})$ in $\mathrm{N}, \mathrm{N}$-dimethylformamide $(10 \mathrm{~mL})$ was 
stirred for $8-16 \mathrm{~h}$ at $70-80^{\circ} \mathrm{C}$. Then the reaction mixture was cooled to temperature and was added water. The aqueous layer was extracted with dichloromethane $(30 \mathrm{~mL} \times 3)$ and the combined organic layer was concentrated under reduced pressure. The residue was solved in ethanol (10 $\mathrm{mL})$ and treated with $80 \%$ hydrazine hydrate $(10.5 \mathrm{mmol})$. The reaction mixture was stirred at reflux for $4-6 \mathrm{~h}$ and concentrated under reduced pressure to give the title compounds $5 \mathbf{b}-\mathbf{c}, \mathbf{6 a}-\mathbf{c}$ and $\mathbf{7 a}-\mathbf{c}$ as yellow oils or off-white solids.

A mixture of pyrrolidin-3-one/piperidin-3-one/piperidin-4-ones (8-10, 15.0 mmol), O-alkylhydroxyl amine hydrochlorides $(18.0 \mathrm{mmol})$ and potassium carbonate $(30.0 \mathrm{mmol})$ in ethanol $(50 \mathrm{~mL}) \mathrm{was}$ stirred for $6-8 \mathrm{~h}$ at $25-50{ }^{\circ} \mathrm{C}$. Then the mixture was cooled to temperature and filtered, the filter cake washed with ethanol. The filtrate was concentrated under reduced pressure to afford the oximes 11-13 as oils. Amine derivatives 14, 15 and 16a-d were prepared from the oximes 11-13 by coupling with $4 \mathbf{b}, \mathbf{c}$ and hydrazinolysis sequentially as described above.

A mixture of 5-formyl-2,4-dimethyl-1H-pyrrole-3-carboxylic acid (17, $3.0 \mathrm{mmol})$, 1-ethyl-3-(3dimethylaminopropyl)carbodiimide hydrochloride (EDCI) $(4.5 \mathrm{mmol})$ and $\mathrm{HOBt}(4.5 \mathrm{mmol})$ in $\mathrm{N}, \mathrm{N}$-Dimethylformamide $(10 \mathrm{~mL})$ was stirred for $0.5 \mathrm{~h}$ at room temperature. Then compounds 5-7, 14-16 or 18-20 (4.5 mmol) and DIEA $(6.6 \mathrm{mmol})$ were added. The reaction mixture was stirred for 12-24 $\mathrm{h}$ and later water was added. The aqueous layer was extracted with dichloromethane/methanol $(10: 1,30 \mathrm{~mL} \times 2)$ and the combined organic layer was concentrated under reduced pressure. A solution of the residue and 5-bromo-1,3-dihydro-2H-pyrrolo[2,3-b]pyridin-2-one (22, $3.0 \mathrm{mmol})$ in ethanol $(10 \mathrm{~mL})$ was stirred at temperature for $8-16 \mathrm{~h}$. The precipitate was filtered, purified via silica gel column chromatography (methanol/dichloromethane 40:1) and recrystallized from ethanol to afford the title compounds $23 \mathbf{a}-\mathbf{q}(26 \%-33 \%$, two steps) as yellow solids.

(Z)-5-[(5-Bromo-2-oxo-1,2-dihydro-3H-pyrrolo[2,3-b]pyridin-3-ylidene)methyl]-2,4-dimethyl-N-[3-(pyrrolidin1-yl)propyl]-1H-pyrrole-3-carboxamide (23a): Yield: 26\%. m.p.: $222-224{ }^{\circ} \mathrm{C} .{ }^{1} \mathrm{H}-\mathrm{NMR}(500 \mathrm{MHz}$, DMSO-d 6 ) $\delta: 13.44(\mathrm{~s}, 1 \mathrm{H}), 11.63(\mathrm{~s}, 1 \mathrm{H}), 8.47(\mathrm{~d}, J=2.0 \mathrm{~Hz}, 1 \mathrm{H}), 8.10(\mathrm{~d}, J=2.0 \mathrm{~Hz}, 1 \mathrm{H}), 7.85(\mathrm{~s}, 1 \mathrm{H})$, $7.74(\mathrm{t}, J=5.5 \mathrm{~Hz}, 1 \mathrm{H}), 3.26(\mathrm{q}, J=12.5 \mathrm{~Hz}, 2 \mathrm{H}), 2.46-2.47(\mathrm{~m}, 6 \mathrm{H}), 2.44(\mathrm{~s}, 3 \mathrm{H}), 2.42(\mathrm{~s}, 3 \mathrm{H}), 1.65-1.71$ $(\mathrm{m}, 6 \mathrm{H}) \mathrm{ppm} .{ }^{13} \mathrm{C}-\mathrm{NMR}\left(400 \mathrm{MHz}, \mathrm{DMSO}-d_{6}\right) \delta: 169.1,164.4,151.1,144.2,137.8,131.8,127.7,126.7$, $126.0,122.2,121.4,112.5,111.0,53.5,53.3,52.0,37.2,28.0,23.0,13.4,10.5,7.2$. MS-ESI $(m / z): 472.6$ $(\mathrm{M}+\mathrm{H})^{+}, 474.6(\mathrm{M}+\mathrm{H})^{+}$.

(Z)-5-[(5-Bromo-2-oxo-1,2-dihydro-3H-pyrrolo[2,3-b]pyridin-3-ylidene)methyl]-2,4-dimethyl-N-[4-(pyrrolidin1-yl)butyl]-1H-pyrrole-3-carboxamide (23b): Yield: 28\%. m.p.: $230-232{ }^{\circ} \mathrm{C} .{ }^{1} \mathrm{H}-\mathrm{NMR}\left(500 \mathrm{MHz}, \mathrm{DMSO}-d_{6}\right)$ $\delta: 13.44(\mathrm{~s}, 1 \mathrm{H}), 11.63(\mathrm{~s}, 1 \mathrm{H}), 8.47(\mathrm{~d}, J=2.0 \mathrm{~Hz}, 1 \mathrm{H}), 8.10(\mathrm{~d}, J=2.0 \mathrm{~Hz}, 1 \mathrm{H}), 7.85(\mathrm{~s}, 1 \mathrm{H}), 7.74(\mathrm{t}$, $J=5.5 \mathrm{~Hz}, 1 \mathrm{H}), 3.22(\mathrm{q}, J=12.5 \mathrm{~Hz}, 2 \mathrm{H}), 2.43(\mathrm{~s}, 3 \mathrm{H}), 2.41(\mathrm{~s}, 3 \mathrm{H}), 2.37-2.40(\mathrm{~m}, 6 \mathrm{H}), 1.65-1.67(\mathrm{~m}, 4 \mathrm{H})$, 1.48-1.53 (m, 4H) ppm. ${ }^{13} \mathrm{C}-\mathrm{NMR}\left(400 \mathrm{MHz}, \mathrm{DMSO}-d_{6}\right) \delta: 169.1,164.3,151.1,144.1,137.6,131.8,127.7$, $126.8,126.0,122.3,121.8,112.5,110.9,55.4,53.6,38.6,27.4,26.0,23.0,13.3,10.5$. MS-ESI $(m / z): 486.6$ $(\mathrm{M}+\mathrm{H})^{+}, 488.6(\mathrm{M}+\mathrm{H})^{+}$.

(Z)-5-[(5-Bromo-2-oxo-1,2-dihydro-3H-pyrrolo[2,3-b]pyridin-3-ylidene)methyl]-2,4-dimethyl-N-[2-(piperidin1-yl)ethyl]-1H-pyrrole-3-carboxamide (23c): Yield: 28\%. m.p.: 239-241 ${ }^{\circ} \mathrm{C} .{ }^{1} \mathrm{H}-\mathrm{NMR}\left(500 \mathrm{MHz}, \mathrm{DMSO}-d_{6}\right)$ $\delta: 13.45(\mathrm{~s}, 1 \mathrm{H}), 11.63(\mathrm{~s}, 1 \mathrm{H}), 8.48(\mathrm{~d}, J=2.0 \mathrm{~Hz}, 1 \mathrm{H}), 8.10(\mathrm{~d}, J=2.0 \mathrm{~Hz}, 1 \mathrm{H}), 7.86(\mathrm{~s}, 1 \mathrm{H}), 7.52(\mathrm{t}$, $J=5.5 \mathrm{~Hz}, 1 \mathrm{H}), 3.34(\mathrm{t}, J=6.5 \mathrm{~Hz}, 2 \mathrm{H}), 2.46(\mathrm{~s}, 3 \mathrm{H}), 2.44-2.45(\mathrm{~m}, 6 \mathrm{H}), 2.44(\mathrm{~s}, 3 \mathrm{H}), 2.17-2.36(\mathrm{~m}, 6 \mathrm{H})$ ppm. ${ }^{13} \mathrm{C}-\mathrm{NMR}\left(400 \mathrm{MHz}, \mathrm{DMSO}-d_{6}\right) \delta: 169.1,164.3,151.1,144.2,138.0,131.8,127.8,126.7,126.0,122.2$, $121.2,112.5,111.1,57.2,53.7,52.0,35.9,25.3,23.7,13.4,10.6,7.2$. MS-ESI $(m / z): 472.6(\mathrm{M}+\mathrm{H})^{+}, 474.6$ $(\mathrm{M}+\mathrm{H})^{+}$.

(Z)-5-[(5-Bromo-2-oxo-1,2-dihydro-3H-pyrrolo[2,3-b]pyridin-3-ylidene)methyl]-2,4-dimethyl-N-[2-(4methylpiperazin-1-yl)ethyl]-1H-pyrrole-3-carboxamide (23d): Yield: 32\%. m.p.: 244-246 ${ }^{\circ} \mathrm{C} .{ }^{1} \mathrm{H}-\mathrm{NMR}$ $\left(500 \mathrm{MHz}, \mathrm{DMSO}-\mathrm{d}_{6}\right) \delta: 13.24(\mathrm{~s}, 1 \mathrm{H}), 9.48(\mathrm{~s}, 1 \mathrm{H}), 8.14(\mathrm{~d}, J=2.0 \mathrm{~Hz}, 1 \mathrm{H}), 7.77(\mathrm{~d}, J=2.0 \mathrm{~Hz}, 1 \mathrm{H}), 7.26$ $(\mathrm{s}, 1 \mathrm{H}), 6.54(\mathrm{t}, J=5.5 \mathrm{~Hz}, 1 \mathrm{H}), 3.55-3.58(\mathrm{~m}, 2 \mathrm{H}), 2.64-2.66(\mathrm{~m}, 4 \mathrm{H}), 2.58(\mathrm{~s}, 3 \mathrm{H}), 2.43-2.50(\mathrm{~m}, 4 \mathrm{H}), 2.37$ $(\mathrm{s}, 3 \mathrm{H}), 2.30$ (s, 3H), 1.60-1.64 (m, 2H) ppm. ${ }^{13} \mathrm{C}-\mathrm{NMR}\left(400 \mathrm{MHz}, \mathrm{DMSO}-d_{6}\right) \delta: 169.1,164.2,151.1,144.1$, 
137.9, 131.7, 127.7, 126.7, 126.0, 122.2, 121.3, 112.5, 111.0, 56.7, 54.8, 52.5, 45.8, 36.1, 13.3, 10.6. MS-ESI $(m / z): 487.7(\mathrm{M}+\mathrm{H})^{+}, 489.7(\mathrm{M}+\mathrm{H})^{+}$.

(S,Z)-5-[(5-Bromo-2-oxo-1,2-dihydro-3H-pyrrolo[2,3-b]pyridin-3-ylidene)methyl]-N-(2-hydroxy-3-

morpholinopropyl)-2,4-dimethyl-1H-pyrrole-3-carboxamide (23e): Yield: $25 \%$. m.p.: 284-286 ${ }^{\circ} \mathrm{C}$. ${ }^{1} \mathrm{H}-\mathrm{NMR}\left(500 \mathrm{MHz}\right.$, DMSO- $\left.d_{6}\right) \delta: 13.45(\mathrm{~s}, 1 \mathrm{H}), 11.64(\mathrm{~s}, 1 \mathrm{H}), 8.48(\mathrm{~d}, J=2.0 \mathrm{~Hz}, 1 \mathrm{H}), 8.10(\mathrm{~d}, J=2.0 \mathrm{~Hz}$, $1 \mathrm{H}), 7.86(\mathrm{~s}, 1 \mathrm{H}), 7.61(\mathrm{t}, J=5.5 \mathrm{~Hz}, 1 \mathrm{H}), 4.76(\mathrm{~s}, 1 \mathrm{H}), 3.78-3.80(\mathrm{~m}, 1 \mathrm{H}), 3.55-3.59(\mathrm{~m}, 4 \mathrm{H}), 3.13-3.18(\mathrm{~m}$, 2H), $2.46(\mathrm{~s}, 3 \mathrm{H}), 2.44(\mathrm{~s}, 3 \mathrm{H}), 2.27-2.36(\mathrm{~m}, 6 \mathrm{H}) \mathrm{ppm} .{ }^{13} \mathrm{C}-\mathrm{NMR}\left(400 \mathrm{MHz}, \mathrm{DMSO}-\mathrm{d}_{6}\right)$ 8: 169.1, 164.6, 151.1, 144.2, 137.9, 131.9, 127.7, 126.7, 126.0, 122.2, 121.3, 112.5, 111.0, 66.7, 66.2, 63.0, 54.0, 43.9, 13.4, 10.6 ppm. MS-ESI $(m / z): 504.6(\mathrm{M}+\mathrm{H})^{+}, 506.6(\mathrm{M}+\mathrm{H})^{+}$.

(Z)-5-[(5-Bromo-2-oxo-1,2-dihydro-3H-pyrrolo[2,3-b]pyridin-3-ylidene)methyl]-2,4-dimethyl-N-[3-(piperidin1-yl)propyll-1H-pyrrole-3-carboxamide (23f): Yield: $27 \%$. m.p.: $236-238{ }^{\circ} \mathrm{C} .{ }^{1} \mathrm{H}-\mathrm{NMR}(500 \mathrm{MHz}$, DMSO-d 6 ) $\delta: 13.44(\mathrm{~s}, 1 \mathrm{H}), 11.64(\mathrm{~s}, 1 \mathrm{H}), 8.47(\mathrm{~d}, J=2.0 \mathrm{~Hz}, 1 \mathrm{H}), 8.10(\mathrm{~d}, J=2.0 \mathrm{~Hz}, 1 \mathrm{H}), 7.85(\mathrm{~s}, 1 \mathrm{H}), 7.56(\mathrm{t}$, $J=6.5 \mathrm{~Hz}, 1 \mathrm{H}), 3.24(\mathrm{q}, J=12.5 \mathrm{~Hz}, 2 \mathrm{H}), 2.44(\mathrm{~s}, 3 \mathrm{H}), 2.42(\mathrm{~s}, 3 \mathrm{H}), 2.17-2.37(\mathrm{~m}, 6 \mathrm{H}), 1.38-1.67(\mathrm{~m}, 8 \mathrm{H})$ ppm. ${ }^{13} \mathrm{C}-\mathrm{NMR}$ (400 MHz, DMSO- $d_{6}$ ) $\delta: 169.1,164.4,151.1,144.1,137.7,131.8,127.7,126.7,126.0,122.2$, 121.6, 112.5, 111.0, 56.3, 54.0, 37.3, 26.5, 25.4, 24.0, 13.3, 10.5 ppm. MS-ESI $(m / z): 486.0(\mathrm{M}+\mathrm{H})^{+}, 488.0$ $(\mathrm{M}+\mathrm{H})^{+}$.

(Z)-5-[(5-Bromo-2-oxo-1,2-dihydro-3H-pyrrolo[2,3-b]pyridin-3-ylidene)methyl]-2,4-dimethyl-N-[4-(piperidin1-yl)butyl]-1H-pyrrole-3-carboxamide (23g): Yield: 33\%. m.p.: $238-240{ }^{\circ} \mathrm{C} .{ }^{1} \mathrm{H}-\mathrm{NMR}\left(500 \mathrm{MHz}, \mathrm{DMSO}-d_{6}\right)$

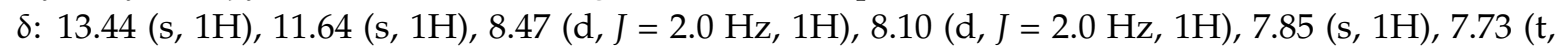
$J=5.5 \mathrm{~Hz}, 1 \mathrm{H}), 3.22(\mathrm{q}, J=12.0 \mathrm{~Hz}, 2 \mathrm{H}), 2.43(\mathrm{~s}, 3 \mathrm{H}), 2.41(\mathrm{~s}, 3 \mathrm{H}), 2.22-2.37(\mathrm{~m}, 6 \mathrm{H}), 1.43-1.53(\mathrm{~m}, 8 \mathrm{H})$, $1.33-1.40(\mathrm{~m}, 2 \mathrm{H}) \mathrm{ppm} .{ }^{13} \mathrm{C}-\mathrm{NMR}\left(400 \mathrm{MHz}\right.$, DMSO- $\left.d_{6}\right) \delta: 169.1,164.4,151.1,144.1,137.6,131.8,127.7$, $126.7,126.0,122.2,121.8,112.5,110.9,58.2,54.0,38.6,27.3,25.4,24.1,23.8,13.2,10.4$ ppm. MS-ESI $(m / z)$ : $500.3(\mathrm{M}+\mathrm{H})^{+}, 502.3(\mathrm{M}+\mathrm{H})^{+}$.

(Z)-5-[(5-Bromo-2-oxo-1,2-dihydro-3H-pyrrolo[2,3-b]pyridin-3-ylidene)methyl]-2,4-dimethyl-N-[3-(4methylpiperazin-1-yl)propyl]-1H-pyrrole-3-carboxamide (23h): Yield: 30\%. m.p.: $238-240{ }^{\circ} \mathrm{C} .{ }^{1} \mathrm{H}-\mathrm{NMR}$ $\left(500 \mathrm{MHz}, \mathrm{DMSO}-d_{6}\right) \delta: 13.44(\mathrm{~s}, 1 \mathrm{H}), 11.62(\mathrm{~s}, 1 \mathrm{H}), 8.47(\mathrm{~d}, J=2.0 \mathrm{~Hz}, 1 \mathrm{H}), 8.10(\mathrm{~d}, J=2.0 \mathrm{~Hz}, 1 \mathrm{H})$, $7.85(\mathrm{~s}, 1 \mathrm{H}), 7.71(\mathrm{t}, J=5.5 \mathrm{~Hz}, 1 \mathrm{H}), 3.22(\mathrm{q}, J=12.5 \mathrm{~Hz}, 2 \mathrm{H}), 2.44(\mathrm{~s}, 3 \mathrm{H}), 2.42(\mathrm{~s}, 3 \mathrm{H}), 2.22-2.35(\mathrm{~m}$, $10 \mathrm{H}), 2.13(\mathrm{~s}, 3 \mathrm{H}), 1.62-1.68(\mathrm{~m}, 2 \mathrm{H}) \mathrm{ppm} .{ }^{13} \mathrm{C}-\mathrm{NMR}\left(400 \mathrm{MHz}, \mathrm{DMSO}-d_{6}\right) \delta: 169.1,164.4,151.1,144.2$, 137.7, 131.8, 127.8, 126.8, 126.0, 122.3, 121.7, 112.6, 111.0, 55.7, 54.7, 52.7, 45.7, 37.3, 26.6, 13.3, 10.5 ppm. MS-ESI $(m / z): 501.3(\mathrm{M}+\mathrm{H})^{+}, 503.3(\mathrm{M}+\mathrm{H})^{+}$.

(Z)-5-[(5-Bromo-2-oxo-1,2-dihydro-3H-pyrrolo[2,3-b]pyridin-3-ylidene)methyl]-2,4-dimethyl-N-[4-(4methylpiperazin-1-yl)butyll-1H-pyrrole-3-carboxamide (23i): Yield: $31 \%$. m.p.: $238-240{ }^{\circ} \mathrm{C} .{ }^{1} \mathrm{H}-\mathrm{NMR}$ $\left(500 \mathrm{MHz}, \mathrm{DMSO}-d_{6}\right) \delta: 13.43(\mathrm{~s}, 1 \mathrm{H}), 11.63(\mathrm{~s}, 1 \mathrm{H}), 8.47(\mathrm{~d}, J=2.0 \mathrm{~Hz}, 1 \mathrm{H}), 8.10(\mathrm{~d}, J=2.0 \mathrm{~Hz}, 1 \mathrm{H}), 7.85$ $(\mathrm{s}, 1 \mathrm{H}), 7.73(\mathrm{t}, J=5.5 \mathrm{~Hz}, 1 \mathrm{H}), 3.22(\mathrm{q}, J=12.0 \mathrm{~Hz}, 2 \mathrm{H}), 2.43(\mathrm{~s}, 3 \mathrm{H}), 2.41(\mathrm{~s}, 3 \mathrm{H}), 2.16-2.37(\mathrm{~m}, 10 \mathrm{H})$, $2.13(\mathrm{~s}, 3 \mathrm{H}), 1.45-1.50(\mathrm{~m}, 4 \mathrm{H}) \mathrm{ppm} .{ }^{13} \mathrm{C}-\mathrm{NMR}\left(400 \mathrm{MHz}\right.$, DMSO- $\left.d_{6}\right) \delta: 169.1,164.4,151.1,144.1,137.6$, 131.8, 127.7, 126.8, 126.0, 122.3, 121.8, 112.5, 110.9, 57.6, 54.8, 52.7, 45.7, 38.6, 27.3, 23.9, 13.3, 10.5 ppm. MS-ESI $(m / z): 515.3(\mathrm{M}+\mathrm{H})^{+}, 517.3(\mathrm{M}+\mathrm{H})^{+}$.

(Z)-5-[(5-Bromo-2-oxo-1,2-dihydro-3H-pyrrolo[2,3-b]pyridin-3-ylidene)methyl]-N-[3-(dimethylamino)propyl]2,4-dimethyl-1H-pyrrole-3-carboxamide (23j): Yield: 29\%. m.p.: $258-260{ }^{\circ} \mathrm{C} .{ }^{1} \mathrm{H}-\mathrm{NMR}(500 \mathrm{MHz}$, DMSO- $\left.d_{6}\right) \delta: 13.44(\mathrm{~s}, 1 \mathrm{H}), 11.61(\mathrm{~s}, 1 \mathrm{H}), 8.47(\mathrm{~d}, J=2.0 \mathrm{~Hz}, 1 \mathrm{H}), 8.10(\mathrm{~d}, J=2.0 \mathrm{~Hz}, 1 \mathrm{H}), 7.85(\mathrm{~s}, 1 \mathrm{H})$, $7.73(\mathrm{t}, J=5.5 \mathrm{~Hz}, 1 \mathrm{H}), 3.25(\mathrm{q}, J=12.5 \mathrm{~Hz}, 2 \mathrm{H}), 2.45(\mathrm{~s}, 3 \mathrm{H}), 2.42(\mathrm{~s}, 3 \mathrm{H}), 2.30(\mathrm{t}, J=6.5 \mathrm{~Hz}, 2 \mathrm{H}), 2.16(\mathrm{~s}$, 6H), 1.62-1.68 (m, 2H) ppm. ${ }^{13} \mathrm{C}-\mathrm{NMR}\left(400 \mathrm{MHz}, \mathrm{DMSO}-d_{6}\right) \delta: 169.1,164.3,151.1,144.1,137.8,131.8$, 127.7, 126.7, 126.0, 122.2, 121.5, 112.5, 111.0, 57.1, 45.2, 37.3, 27.1, 13.3, 10.5 ppm. MS-ESI $(\mathrm{m} / \mathrm{z}): 446.2$ $(\mathrm{M}+\mathrm{H})^{+}, 448.2(\mathrm{M}+\mathrm{H})^{+}$.

(Z)-5-[(5-Bromo-2-oxo-1,2-dihydro-3H-pyrrolo[2,3-b]pyridin-3-ylidene)methyl]-N-[3-(diethylamino)propyl]2,4-dimethyl-1H-pyrrole-3-carboxamide (23k): Yield: $30 \%$. m.p.: $234-236{ }^{\circ} \mathrm{C} .{ }^{1} \mathrm{H}-\mathrm{NMR}(500 \mathrm{MHz}$, DMSO- $\left.d_{6}\right) \delta: 13.45(\mathrm{~s}, 1 \mathrm{H}), 11.63(\mathrm{~s}, 1 \mathrm{H}), 8.47(\mathrm{~d}, J=2.0 \mathrm{~Hz}, 1 \mathrm{H}), 8.11(\mathrm{~d}, J=2.0 \mathrm{~Hz}, 1 \mathrm{H}), 7.86(\mathrm{~s}, 1 \mathrm{H})$, 
$7.74(\mathrm{t}, J=5.5 \mathrm{~Hz}, 1 \mathrm{H}), 3.25-3.31(\mathrm{~m}, 2 \mathrm{H}), 3.06-3.15(\mathrm{~m}, 6 \mathrm{H}), 2.47(\mathrm{~s}, 3 \mathrm{H}), 2.44(\mathrm{~s}, 3 \mathrm{H}), 1.86-1.92(\mathrm{~m}, 2 \mathrm{H})$, $1.21(\mathrm{t}, J=7.2 \mathrm{~Hz}, 6 \mathrm{H}) \mathrm{ppm} .{ }^{13} \mathrm{C}-\mathrm{NMR}\left(400 \mathrm{MHz}, \mathrm{DMSO}-d_{6}\right) \delta: 169.1,164.8,151.2,144.2,137.9,131.8$, 127.8, 126.8, 126.0, 122.2, 121.1, 112.5, 111.2, 48.4, 46.0, 36.0, 23.5, 13.4, 10.6, 8.4 ppm. MS-ESI $(\mathrm{m} / \mathrm{z})$ : $474.3(\mathrm{M}+\mathrm{H})^{+}, 476.3(\mathrm{M}+\mathrm{H})^{+}$.

5-[(Z)-(5-Bromo-2-oxo-1,2-dihydro-3H-pyrrolo[2,3-b]pyridin-3-ylidene)methyl]-N-\{3-[(E)-3-(methoxyimino) pyrrolidin-1-yl]propyl\}-2,4-dimethyl-1H-pyrrole-3-carboxamide (231): Yield: $27 \%$. m.p.: $226-228{ }^{\circ} \mathrm{C}$. ${ }^{1} \mathrm{H}-\mathrm{NMR}\left(500 \mathrm{MHz}\right.$, DMSO- $\left.d_{6}\right) \delta: 13.45(\mathrm{~s}, 1 \mathrm{H}), 11.63(\mathrm{~s}, 1 \mathrm{H}), 8.47(\mathrm{~d}, J=2.0 \mathrm{~Hz}, 1 \mathrm{H}), 8.10(\mathrm{~d}, J=2.0 \mathrm{~Hz}$, $1 \mathrm{H}), 7.85(\mathrm{~s}, 1 \mathrm{H}), 7.75(\mathrm{t}, J=5.5 \mathrm{~Hz}, 1 \mathrm{H}), 3.95-4.10(\mathrm{~m}, 4 \mathrm{H}), 3.78(\mathrm{~s}, 3 \mathrm{H}), 3.48-3.56(\mathrm{~m}, 2 \mathrm{H}), 3.28-3.32$ $(\mathrm{m}, 2 \mathrm{H}), 2.63-2.69(\mathrm{~m}, 2 \mathrm{H}), 2.44(\mathrm{~s}, 3 \mathrm{H}), 2.42(\mathrm{~s}, 3 \mathrm{H}), 1.80-1.85(\mathrm{~m}, 2 \mathrm{H}) \mathrm{ppm} .{ }^{13} \mathrm{C}-\mathrm{NMR}(400 \mathrm{MHz}$, DMSO- $d_{6}$ ) $\delta: 169.1,164.5,154.1,151.1,144.2,137.7,131.8,127.7,126.7,126.0,122.2,121.4,112.5,111.0$, $62.7,61.4,45.6,43.8,35.6,28.9,18.5,13.3,10.5$ ppm. MS-ESI $(m / z): 515.1(\mathrm{M}+\mathrm{H})^{+}, 517.1(\mathrm{M}+\mathrm{H})^{+}$.

5-[(Z)-(5-Bromo-2-oxo-1,2-dihydro-3H-pyrrolo[2,3-b]pyridin-3-ylidene)methyl]-N-\{2-[(E)-3-(methoxyimino) piperidin-1-yl]ethyl\}-2,4-dimethyl-1H-pyrrole-3-carboxamide (23m): Yield: $26 \%$. m.p.: $198-200{ }^{\circ} \mathrm{C}$. ${ }^{1} \mathrm{H}-\mathrm{NMR}\left(500 \mathrm{MHz}\right.$, DMSO- $\left.d_{6}\right) \delta: 13.45(\mathrm{~s}, 1 \mathrm{H}), 11.61(\mathrm{~s}, 1 \mathrm{H}), 8.47(\mathrm{~d}, J=2.0 \mathrm{~Hz}, 1 \mathrm{H}), 8.10(\mathrm{~d}, J=2.0 \mathrm{~Hz}$, $1 \mathrm{H}), 7.85(\mathrm{~s}, 1 \mathrm{H}), 7.80(\mathrm{t}, J=5.5 \mathrm{~Hz}, 1 \mathrm{H}), 3.98-4.24(\mathrm{~m}, 4 \mathrm{H}), 3.74(\mathrm{~s}, 3 \mathrm{H}), 3.46-3.48(\mathrm{~m}, 4 \mathrm{H}), 2.44(\mathrm{~s}, 3 \mathrm{H})$, $2.42(\mathrm{~s}, 3 \mathrm{H}), 2.22-2.36(\mathrm{~m}, 2 \mathrm{H}), 1.63-1.69(\mathrm{~m}, 2 \mathrm{H}) \mathrm{ppm} .{ }^{13} \mathrm{C}-\mathrm{NMR}\left(400 \mathrm{MHz}, \mathrm{DMSO}-d_{6}\right) \delta: 169.1,164.6$, 153.9, 151.1, 144.2, 137.8, 131.9, 127.8, 126.8, 126.0, 122.2, 121.2, 112.5, 111.1, 63.8, 61.0, 56.0, 43.3, 38.2, 22.8, 18.5, 13.3, 10.4 ppm. MS-ESI $(m / z): 515.3(\mathrm{M}+\mathrm{H})^{+}, 517.3(\mathrm{M}+\mathrm{H})^{+}$.

(Z)-5-[(5-Bromo-2-oxo-1,2-dihydro-3H-pyrrolo[2,3-b]pyridin-3-ylidene)methyl]-N-\{2-[4-(methoxyimino) piperidin-1-yl]ethyl\}-2,4-dimethyl-1H-pyrrole-3-carboxamide (23n): Yield: $29 \%$. m.p.: 262-264 ${ }^{\circ} \mathrm{C} .{ }^{1} \mathrm{H}-\mathrm{NMR}$ $\left(500 \mathrm{MHz}\right.$, DMSO- $\left.d_{6}\right) \delta: 13.44(\mathrm{~s}, 1 \mathrm{H}), 11.62(\mathrm{~s}, 1 \mathrm{H}), 8.46(\mathrm{~d}, J=2.0 \mathrm{~Hz}, 1 \mathrm{H}), 8.10(\mathrm{~d}, J=2.0 \mathrm{~Hz}, 1 \mathrm{H}), 7.85$ $(\mathrm{s}, 1 \mathrm{H}), 7.58(\mathrm{t}, J=5.5 \mathrm{~Hz}, 1 \mathrm{H}), 3.71(\mathrm{~s}, 3 \mathrm{H}), 3.33-3.39(\mathrm{~m}, 2 \mathrm{H}), 2.56(\mathrm{t}, J=6.0 \mathrm{~Hz}, 2 \mathrm{H}), 2.47-2.52(\mathrm{~m}, 6 \mathrm{H})$, $2.46(\mathrm{~s}, 3 \mathrm{H}), 2.44(\mathrm{~s}, 3 \mathrm{H}), 2.23(\mathrm{t}, J=6.0 \mathrm{~Hz}, 2 \mathrm{H}) \mathrm{ppm} .{ }^{13} \mathrm{C}-\mathrm{NMR}\left(400 \mathrm{MHz}, \mathrm{DMSO}-d_{6}\right) \delta: 169.1,164.2$, 156.3, 151.1, 144.2, 137.9, 131.8, 127.7, 126.7, 126.0, 122.2, 121.4, 112.5, 111.0, 60.6, 56.2, 53.1, 51.7, 36.4, 30.8, 24.9, 13.3, 10.5 ppm. MS-ESI $(m / z): 515.2(\mathrm{M}+\mathrm{H})^{+}, 517.3(\mathrm{M}+\mathrm{H})^{+}$.

(Z)-5-[(5-Bromo-2-oxo-1,2-dihydro-3H-pyrrolo[2,3-b]pyridin-3-ylidene)methyl]-N-\{3-[4-(methoxyimino) piperidin-1-yl]propyl\}-2,4-dimethyl-1H-pyrrole-3-carboxamide (23o): Yield: $32 \%$. m.p.: $233-235{ }^{\circ} \mathrm{C}$. ${ }^{1} \mathrm{H}-\mathrm{NMR}\left(500 \mathrm{MHz}\right.$, DMSO- $\left.d_{6}\right) \delta: 13.44(\mathrm{~s}, 1 \mathrm{H}), 11.63(\mathrm{~s}, 1 \mathrm{H}), 8.47(\mathrm{~d}, J=2.0 \mathrm{~Hz}, 1 \mathrm{H}), 8.10(\mathrm{~d}, J=2.0 \mathrm{~Hz}$, $1 \mathrm{H}), 7.85(\mathrm{~s}, 1 \mathrm{H}), 7.71(\mathrm{t}, J=5.5 \mathrm{~Hz}, 1 \mathrm{H}), 3.71(\mathrm{~s}, 3 \mathrm{H}), 3.25(\mathrm{q}, J=12.5 \mathrm{~Hz}, 2 \mathrm{H}), 2.45-2.49(\mathrm{~m}, 6 \mathrm{H}), 2.44(\mathrm{~s}$, $3 \mathrm{H}), 2.42(\mathrm{~s}, 3 \mathrm{H}), 2.38(\mathrm{t}, J=5.9 \mathrm{~Hz}, 2 \mathrm{H}), 2.22(\mathrm{t}, J=7.0 \mathrm{~Hz}, 2 \mathrm{H}), 1.65-1.71(\mathrm{~m}, 2 \mathrm{H}) \mathrm{ppm} .{ }^{13} \mathrm{C}-\mathrm{NMR}$ (400 MHz, DMSO- $\left.d_{6}\right) \delta: 169.1,164.4,156.4,151.1,144.2,137.7,131.8,127.7,126.8,126.0,122.2,121.6$, 112.5, 111.0, 60.5, 55.0, 53.3, 52.0, 37.2, 30.7, 26.9, 24.8, 13.3, 10.5 ppm. MS-ESI $(m / z): 529.3(\mathrm{M}+\mathrm{H})^{+}$, $531.3(\mathrm{M}+\mathrm{H})^{+}$.

(Z)-5-[(5-Bromo-2-oxo-1,2-dihydro-3H-pyrrolo[2,3-b]pyridin-3-ylidene)methyl]-N-\{3-[4-(ethoxyimino) piperidin-1-yl]propyl\}-2,4-dimethyl-1H-pyrrole-3-carboxamide (23p): Yield: $31 \%$. m.p.: 237-239 ${ }^{\circ} \mathrm{C}$. ${ }^{1} \mathrm{H}-\mathrm{NMR}\left(500 \mathrm{MHz}\right.$, DMSO- $\left.d_{6}\right) \delta: 13.44(\mathrm{~s}, 1 \mathrm{H}), 11.63(\mathrm{~s}, 1 \mathrm{H}), 8.47(\mathrm{~d}, J=2.0 \mathrm{~Hz}, 1 \mathrm{H}), 8.10(\mathrm{~d}, J=2.0 \mathrm{~Hz}$, $1 \mathrm{H}), 7.85(\mathrm{~s}, 1 \mathrm{H}), 7.71(\mathrm{t}, J=5.5 \mathrm{~Hz}, 1 \mathrm{H}), 3.97(\mathrm{q}, J=7.0 \mathrm{~Hz}, 2 \mathrm{H}), 3.25(\mathrm{q}, J=12.5 \mathrm{~Hz}, 2 \mathrm{H}), 2.45-2.48(\mathrm{~m}$, $6 \mathrm{H}), 2.44(\mathrm{~s}, 3 \mathrm{H}), 2.42(\mathrm{~s}, 3 \mathrm{H}), 2.37-2.40(\mathrm{~m}, 2 \mathrm{H}), 2.22-2.24(\mathrm{~m}, 2 \mathrm{H}), 1.65-1.71(\mathrm{~m}, 2 \mathrm{H}), 1.16(\mathrm{t}, J=7.0 \mathrm{~Hz}$, 3H) ppm. ${ }^{13} \mathrm{C}-\mathrm{NMR}\left(400 \mathrm{MHz}\right.$, DMSO- $\left.d_{6}\right) \delta: 169.1,164.4,156.0,151.1,144.1,137.7,131.8,127.7,126.7$, 126.0, 122.2, 121.6, 112.5, 111.0, 67.8, 55.0, 53.4, 52.0, 37.2, 30.8, 26.9, 24.9, 14.5, 13.3, 10.5 ppm. MS-ESI $(m / z): 543.3(\mathrm{M}+\mathrm{H})^{+}, 545.3(\mathrm{M}+\mathrm{H})^{+}$.

(Z)-N-\{3-[4-(Benzyloxyimino)piperidin-1-yl]propyl\}-5-[(5-bromo-2-oxo-1,2-dihydro-3H-pyrrolo[2,3-b]pyridin3-ylidene)methyl)]-2,4-dimethyl-1H-pyrrole-3-carboxamide (23q): Yield: $30 \%$. m.p.: $226-228{ }^{\circ} \mathrm{C} .{ }^{1} \mathrm{H}-\mathrm{NMR}$ $\left(500 \mathrm{MHz}\right.$, DMSO- $\left.d_{6}\right) \delta: 13.44(\mathrm{~s}, 1 \mathrm{H}), 11.63(\mathrm{~s}, 1 \mathrm{H}), 8.47(\mathrm{~d}, J=2.0 \mathrm{~Hz}, 1 \mathrm{H}), 8.10(\mathrm{~d}, J=2.0 \mathrm{~Hz}, 1 \mathrm{H}), 7.85$ $(\mathrm{s}, 1 \mathrm{H}), 7.71(\mathrm{t}, J=5.5 \mathrm{~Hz}, 1 \mathrm{H}), 7.27-7.36(\mathrm{~m}, 5 \mathrm{H}), 4.99(\mathrm{~s}, 2 \mathrm{H}), 3.25(\mathrm{q}, J=12.5 \mathrm{~Hz}, 2 \mathrm{H}), 2.51-2.53(\mathrm{~m}$, $2 \mathrm{H}), 2.47-2.48(\mathrm{~m}, 4 \mathrm{H}), 2.44(\mathrm{~s}, 3 \mathrm{H}), 2.42(\mathrm{~s}, 3 \mathrm{H}), 2.37-2.40(\mathrm{~m}, 2 \mathrm{H}), 2.22(\mathrm{t}, J=5.8 \mathrm{~Hz}, 2 \mathrm{H}), 1.65-1.71(\mathrm{~m}$, 2H) ppm. ${ }^{13} \mathrm{C}-\mathrm{NMR}\left(400 \mathrm{MHz}\right.$, DMSO- $\left.d_{6}\right) \delta: 169.1,164.4,157.2,151.1,144.1,138.2,137.7,131.8,128.2$, 
127.7, 127.5, 126.7, 126.0, 122.2, 121.6, 112.5, 111.0, 74.3, 55.0, 53.3, 52.0, 37.2, 30.8, 26.9, 25.0, 13.3, 10.5 ppm. MS-ESI $(m / z): 605.3(\mathrm{M}+\mathrm{H})^{+}, 607.3(\mathrm{M}+\mathrm{H})^{+}$.

\section{Conclusions}

In summary, a series of novel 5-bromo-7-azaindolin-2-one derivatives containing a 2,4-dimethyl-1H-pyrrole-3-carboxamide moiety were designed, synthesized and evaluated for their in vitro antitumor activity by MTT assay. Our results reveal that many target compounds exhibit broad-spectrum antitumor potency which is better than Sunitinib. The most active compound 23p $\left(\mathrm{IC}_{50}\right.$ : 2.357-3.012 $\left.\mu \mathrm{M}\right)$ was found 11.3- to 8.4-fold more potent than Sunitinib against all of the tested cell lines, HepG2, A549 and Skov-3, respectively. Studies to determine the in vivo pharmacokinetics and efficacy of compounds 23c, 23d and 23p against some selected tumor cell lines are currently underway.

Acknowledgments: This work was supported by National Key Research and Development Program (2016YFA0201500), the National S\&T Major Special Project on Major New Drug Innovations (No. 162 2014ZX09507009-003, No. 2015ZX09102007-008) and NSFC (No. 163 81373267, No. 21502237).

Author Contributions: Conceived of and designed the experiments: Yun Chai, Huiyuan Guo, and Mingliang Liu. Performed the experiments: Jun Zhang, Weiyi Shen and Xiaoning Li. Analyzed the data: Senjun Li and Kai Lv. Wrote the paper: Yun Chai, Mingliang Liu and Jun Zhang. All authors read and approved the final manuscript.

Conflicts of Interest: The authors declare no conflict of interest.

\section{References}

1. Kassem, M.G.; Motiur Rahman, A.F.M.; Korashy, H.M. Sunitinib malate. Profiles Drug Subst. Excip. Relat. Methodol. 2012, 37, 363-388. [PubMed]

2. Kulke, M.H.; Bendell, J.; Kvols, L.; Picus, J.; Pommier, R.; Yao, J. Evolving Diagnostic and Treatment Strategies for Pancreatic Neuroendocrine Tumors. J. Hematol. Oncol. 2011, 4, 29. [CrossRef] [PubMed]

3. Norden, A.D.; Drappatz, J.; Wen, P.Y. Targeted drug therapy for meningiomas. Neurosurg. Focus 2007, 23, E12. [CrossRef] [PubMed]

4. Houk, B.E.; Bello, C.L.; Poland, B.; Rosen, L.S.; Demetri, G.D.; Motzer, R.J. Relationship between exposure to sunitinib and efficacy and tolerability endpoints in patients with cancer: Results of a pharmacokinetic/pharmacodynamic meta-analysis. Cancer Chemother. Pharmacol. 2010, 66, 357-371. [CrossRef] [PubMed]

5. Novello, S.; Scagliotti, G.V.; Rosell, R.; Socinski, M.A.; Brahmer, J.; Atkins, J.; Pallares, C.; Burgess, R.; Tye, L.; Selaru, P.; et al. Phase II study of continuous daily sunitinib dosing in patients with previously treated advanced non-small cell lung cancer. Br. J. Cancer 2009, 101, 1543-1548. [CrossRef] [PubMed]

6. Islam, I.; Bryant, J.; Chou, Y.L.; Kochanny, M.J.; Lee, W.; Phillips, G.B.; Yu, H.Y.; Adler, M.; Whitlow, M.; Ho, E.; et al. Indolinone based phosphoinositide-dependent kinase-1 (PDK1) inhibitors. Part 1: Design, synthesis and biological activity. Bioorg. Med. Chem. Lett. 2007, 17, 3814-3818. [CrossRef] [PubMed]

7. Islam, I.I.; Brown, G.; Bryant, J.; Hrvatin, P.; Kochanny, M.J.; Phillips, G.B.; Yuan, S.D.; Adler, M.; Whitlow, M.; Lentz, D.; et al. Indolinone based phosphoinositide-dependent kinase-1 (PDK1) inhibitors. Part 2: Optimization of BX-517. Bioorg. Med. Chem. Lett. 2007, 17, 3819-3825. [CrossRef] [PubMed]

8. Tang, P.C.; Su, Y.D.; Feng, J.; Fu, J.H.; Yang, J.L.; Xiao, L.; Peng, J.H.; Li, Y.L.; Zhang, L.; Hu, B.; et al. Novel potent orally active multitargeted receptor tyrosine kinase inhibitors: Synthesis, structure-activity relationships, and antitumor activities of 2-indolinone derivatives. J. Med. Chem. 2010, 53, 8140-8149. [CrossRef] [PubMed]

9. Lu, H.Y.; Lin, C.; Zheng, Z.B.; Li, S.; Guo, S.X.; Zhang, X.Y.; Guo, S.; Zhang, X.Y.; Fu, M.; Liang, X.; et al. Angiogenesis Inhibitor Z24 Induces Endothelial Cell Apoptosis and Suppresses Tumor Growth and Metastasis. J. Pharmacol. Sci. 2005, 97, 533-540. [CrossRef] [PubMed] 
10. Sun, L.; Liang, C.; Shirazian, S.; Zhou, Y.; Miller, T.; Cui, J.; Fukuda, J.Y.; Chu, J.Y.; Nematalla, A.; Wang, X.Y.; et al. Discovery of 5-[5-Fluoro-2-oxo-1,2-dihydroindol-(3Z)-ylidenemethyl]-2,4-dimethyl1H-pyrrole-3-carboxylic Acid (2-Diethylaminoethyl)amide, a Novel Tyrosine Kinase Inhibitor Targeting Vascular Endothelial and Platelet-Derived Growth Factor Receptor Tyrosine Kinase. J. Med. Chem. 2003, 46, 1116-1119. [PubMed]

11. Manley, J.M.; Kalman, M.J.; Conway, B.G.; Ball, C.C.; Havens, J.L.; Vaidyannathan, R. Early amidation approach to 3-[(4-amido)pyrrol-2-yl]-2-indolinones. J. Med. Chem. 2003, 68, 6447-6450. [CrossRef] [PubMed]

12. Lv, K.; Wang, L.L.; Liu, M.L.; Zhou, X.B.; Fan, S.Y.; Liu, H.Y.; Zheng, Z.B.; Li, S. Synthesis and antitumor activity of 5-[1-(3-(dimethylamino)propyl)-5-halogenated-2-oxoindolin-(3Z)-ylidenemethyl]-2,4-dimethyl-1Hpyrrole-3-carboxamides. Bioorg. Med. Chem. Lett. 2011, 21, 3062-3065. [CrossRef] [PubMed]

13. Lv, K.; Wang, L.L.; Zhou, X.B.; Liu, M.L.; Liu, H.Y.; Zheng, Z.B.; Li, S. Synthesis and in vitro antitumor activity of 1-(3-dimethylamino)propyl indolin-2-one derivatives. Med. Chem. Res. 2013, 22, 1723-1729. [CrossRef]

14. Wang, M.; Ye, C.; Liu, M.L.; Wu, Z.Y.; Li, L.H.; Wang, C.L.; Liu, X.J.; Guo, H.Y. Synthesis and antitumor activity of 5-(5-halogenated-2-oxo-1H-pyrrolo[2,3-b]pyridin-(3Z)-ylidenemethyl)-2,4-dimethyl-1H-pyrrole-3carboxamides. Bioorg. Med. Chem. Lett. 2015, 25, 2782-2787. [CrossRef] [PubMed]

15. Tang, J.; Wang, B.X.; Wu, T.; Wan, J.T.; Tu, Z.C.; Njire, M.; Wan, B.J.; Franzblauc, S.G.; Zhang, T.Y.; Lu, X.Y.; et al. Design, Synthesis, and Biological Evaluation of Pyrazolo[1,5-a]pyridine-3-carboxamides as Novel Antitubercular Agents. ACS Med. Chem. Lett. 2015, 6, 814-818. [CrossRef] [PubMed]

16. Green, L.M.; Reade, J.L.; Ware, C.F. Rapid colormetric assay for cell viability: Application to the quantitation of cytotoxic and growth inhibitory lymphokines. J. Immunol. Methods 1984, 70, 257-268. [CrossRef]

17. Lv, K.; Sun, Y.X.; Sun, L.Y.; Wei, Z.Q.; Guo, H.Y.; Wu, J.W.; Liu, H.Y. Design, Synthesis, and in vitro Antibacterial Activity of Fluoroquinolone Derivatives Containing a Chiral 3-(Alkoxyimino)-2-(aminomethyl) azetidine Moiety. ChemMedChem 2012, 7, 1230-1236. [CrossRef] [PubMed]

18. Wang, X.Y.; Guo, Q.; Wang, Y.C.; Liu, B.Q.; Liu, M.L.; Sun, L.Y.; Guo, H.Y. Synthesis and antibacterial activity of 7-(3-amino-4-alkoxyimino-1-piperidyl)-quinolones. Acta Pharm. Sin. 2008, 43, 819-827.

19. Jia, X.D.; Wang, S.; Wang, M.H.; Liu, M.L.; Xia, G.M.; Liu, X.J.; Chai, Y.; He, H.W. Synthesis and in vitro antitumor activity of novel naphthyridinone derivatives. Chin. Chem. Lett. 2016. [CrossRef]

Sample Availability: Samples of the compounds $\mathbf{2 3 f}-\mathbf{q}$ are available from the authors.

(C) 2016 by the authors; licensee MDPI, Basel, Switzerland. This article is an open access article distributed under the terms and conditions of the Creative Commons Attribution (CC-BY) license (http:/ / creativecommons.org/licenses/by/4.0/). 